Yiwei Wang

Key Laboratory for Mechanics in Fluid Solid

Coupling Systems,

Institute of Mechanics,

Chinese Academy of Sciences,

No. 15 Beisihuanxi Road,

Beijing 100190, China

e-mail: wangyw@imech.ac.cn

Chenguang Huang

Key Laboratory for Mechanics in Fluid Solid

Coupling Systems,

Institute of Mechanics,

Chinese Academy of Sciences,

No. 15 Beisihuanxi Road,

Beijing 100190, China

e-mail: huangcg@imech.ac.cn

Xin Fang

The State Key Laboratory of Nonlinear Mechanics, Institute of Mechanics,

Chinese Academy of Sciences,

No. 15 Beisihuanxi Road,

Beijing 100190, China

Xianian Yu

Key Laboratory for Mechanics in Fluid Solid

Coupling Systems,

Institute of Mechanics,

Chinese Academy of Sciences,

No. 15 Beisihuanxi Road

Beijing 100190, China

Xiaocui Wu

Key Laboratory for Mechanics in Fluid Solid

Coupling Systems,

Institute of Mechanics,

Chinese Academy of Sciences,

No. 15 Beisihuanxi Road,

Beijing 100190, China

Tezhuan Du

Key Laboratory for Mechanics in Fluid Solid

Coupling Systems,

Institute of Mechanics,

Chinese Academy of Sciences,

No. 15 Beisihuanxi Road,

Beijing 100190, China

\title{
Cloud Cavitating Flow Over a Submerged Axisymmetric Projectile and Comparison Between Two-Dimensional RANS and Three-Dimensional Large-Eddy Simulation Methods
}

For the cloud cavitation around slender axisymmetric projectiles, a two-dimensional (2D) numerical method was based on the mixture approach with Singhal cavitation model and modified renormalization-group $(R N G) k-\varepsilon$ turbulence model, and a threedimensional (3D) method was established with large-eddy simulation (LES) and volume of fraction (VOF) approach. The commercial computational fluid dynamic (CFD) software FLUENT is used for the $2 D$ simulation, and the open source code OpenFOAM is adopted for the $3 D$ calculation. Experimental and numerical results were presented on a typical case, in which the projectile moves with a quasi-constant axial speed. Simulation results agree well with experimental results. An analysis of the evolution of cavitating flow was performed, and the related physical mechanism was discussed. Results demonstrate that shedding cavity collapse plays an important role in the generation and acceleration of re-entry jet, which is the main reason for the instability of cloud cavitation. The 2D Reynolds-Averaged Navier-Stokes (RANS) method can represent the physical phenomena effectively. The $3 D$ LES method can give an efficient simulation on the shedding vortices, and considerable accurate shapes of shedding cavities are captured. [DOI: $10.1115 / 1.4032293$ ]

\section{Introduction}

When vehicles run in water with a high-speed, a part of the liquid water is transformed into vapor in the low-pressure regions around the body as the cavitation phenomena [1]. Such phenomena have several forms, and cloud cavitation is one of the most complex ones. Vortices and bubbles shed massively into the downstream flow field with certain frequencies to induce noticeable changes in the surface pressure. These unsteady cavitation phenomena are critical for the efficient design and improved performance of underwater vehicles.

Contributed by the Fluids Engineering Division of ASME for publication in the Journal of Fluids Engineering. Manuscript received April 7, 2015; final manuscript received November 12, 2015; published online February 17, 2016. Assoc. Editor: Samuel Paolucci.

Experiment and numerical simulation are the main approaches to study the unsteady behavior and instability of cloud cavitation. Experimental research is mostly conducted in cavitation water tunnels, and the flow motion and structure are investigated by using a high-speed camera, particle-image velocimetry, and X-ray [2-4]. In previous works, re-entry jet has been found as the key factor to induce the instability. Summarizations and classifications have been conducted on the cavitating flow around hydrofoils [5]. CFD method is mostly used for numerical simulations. Navier-Stokes equations with single fluid/multiple phases are often adopted as the governing equations, and cavitation models are introduced to calculate the mass transfer between water and vapor phases. Common cavitation models include Singhal model [6] based on Rayleigh-Plesset equation and Kunz model [7] from Ginzburg-Landau potential [8]. 


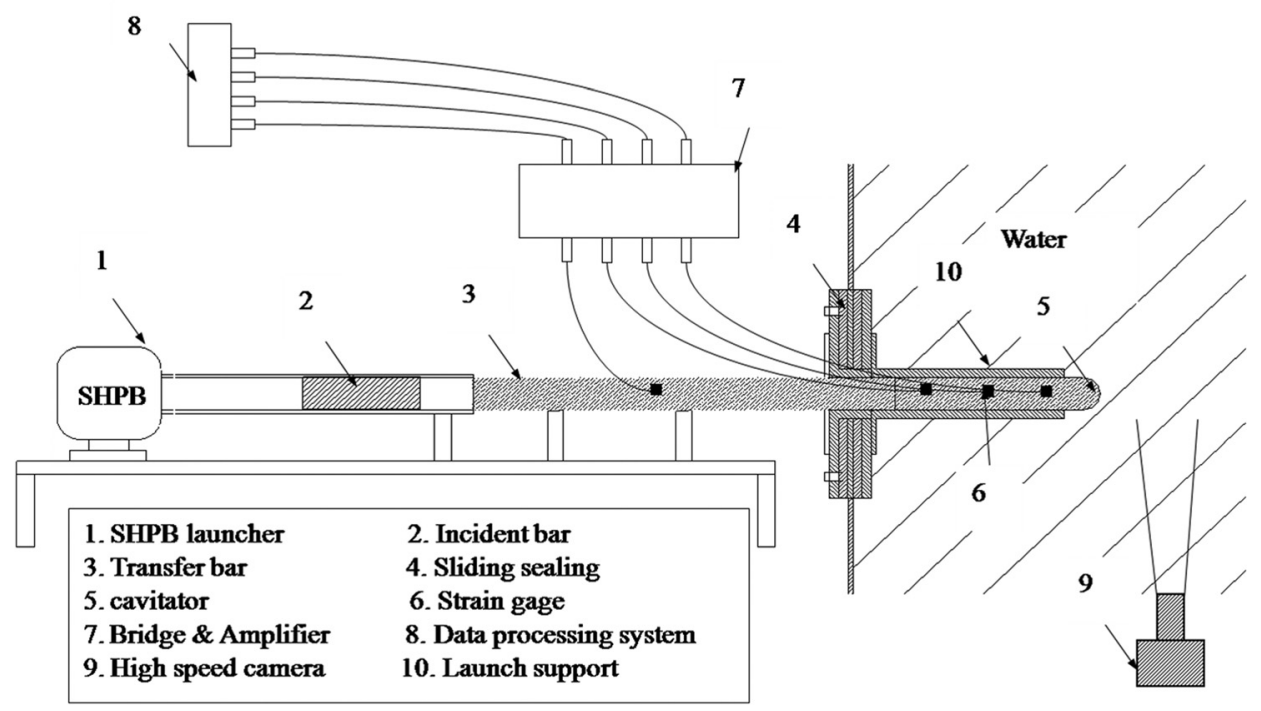

Fig. 1 Underwater launch system: 1-SHPB launcher, 2-incident bar, 3-transfer bar, 4sliding scale, 5-cavitator, 6-strain gauge, 7-bridge and amplifier, 8-data-processing system, 9-high-speed camera, and 10-launch support

Owing to the close relation between the unsteady behavior of cloud cavities and the motion of vortices [9], the simulation method on turbulence is a key issue. Solving RANS equations with turbulence models and modifications has been the main way for engineering applications. For example, Zhou and Wang [10] used the standard RNG $k-\varepsilon$ turbulence model for stable cavities and the modified RNG $k-\varepsilon$ model for unstable cavity shedding; the agreements between numerical and experimental results were presented. Coutier-Delgosha et al. [11-14] published a series of papers that presented a new single-fluid approach, physical modifications of RNG $k-\varepsilon$ turbulence model, and other numerical methods about cloud cavitation to investigate the unsteady characteristics of cavitating flow around hydrofoils and in venture-type sections. Wu et al. established a filter-based $k-\varepsilon$ model to maintain large-scale flow characteristics and avoid excessive dissipation in small-scale motion [15]. Similar approaches, such as partially averaged Navier-Stokes method [16-20], are also widely used.

LES method is also adopted recently for the research of cavitating flows around hydrofoil and propeller, which can capture considerable details of the flow field and give good predictions of large-scale turbulent eddies with high accuracy. Some promising results have been published [21-28]. However, LES method remains difficult to apply to complex engineering problems because of the necessary large mesh size and computation scale. Therefore, comparison of simulation capability between LES and modified RANS methods is needed, which can provide a good basis for later applications.

A split Hopkinson pressure bar (SHPB) launching system is established as an experimental method in this study to investigate the unsteady characteristics of cloud cavitations around axisymmetric projectiles and compare different approaches on turbulence. On the basis of the mixture approach with modified RNG $k-\varepsilon$ turbulence model and the VOF approach with LES solver, 2D and $3 \mathrm{D}$ numerical methods are both used. Through comparisons and analyses of the results obtained from the two methods, we investigate the evolution process and characteristics and discuss the mechanism.

\section{Experimental Setup}

2.1 Experimental System Principle. SHPB technique is widely applied to measure the dynamic mechanical properties of materials. By the stress wave generated by SHPB, the experimental system can accelerate transiently the body to $30 \mathrm{~m} / \mathrm{s}$ in less than $200 \mu$ s, with slight disturbance during the entire process. On the basis of a previous investigation [29], an SHPB launching system is adopted in this study.

2.2 System Components. As shown in Fig. 1, the system mainly consists of four parts, namely, launch system (1-3 and 5), water tank (4 and 10), stain test system (6-8), and high-speed camera (9). The tank dimensions are $C \times B \times H=2000 \mathrm{~mm}$ $\times 1000 \mathrm{~mm} \times 800 \mathrm{~mm}$, and the top of the tank is directly exposed to open air (as shown in Fig. 2).

2.3 Projectile Model and Typical Experimental Condition. The projectile model (as shown in Fig. 3) is a slender cylinder with a conical head, which is initially placed on a support in the middle of the test section. Its material is a polished stainless steel. The total length is $246 \mathrm{~mm}$, and the diameter is $37 \mathrm{~mm}$. The conical angle is $90 \mathrm{deg}$. In a typical experiment, the analysis of obtained images indicates that the speed is approximately uniform at $18.5 \mathrm{~m} / \mathrm{s}$. The temperature is set as $20^{\circ} \mathrm{C}$. The cavitation number can be calculated as

$$
\sigma=\frac{p_{\infty}-p_{v}\left(20^{\circ} \mathrm{C}\right)}{\frac{1}{2} \rho v_{0}^{2}}=0.66
$$

where $p_{\infty}$ is the pressure in open air, $p_{v}\left(20^{\circ} \mathrm{C}\right)$ is the saturated vapor pressure at $20^{\circ} \mathrm{C}, \rho$ is the water density, and $v_{0}$ is the speed.

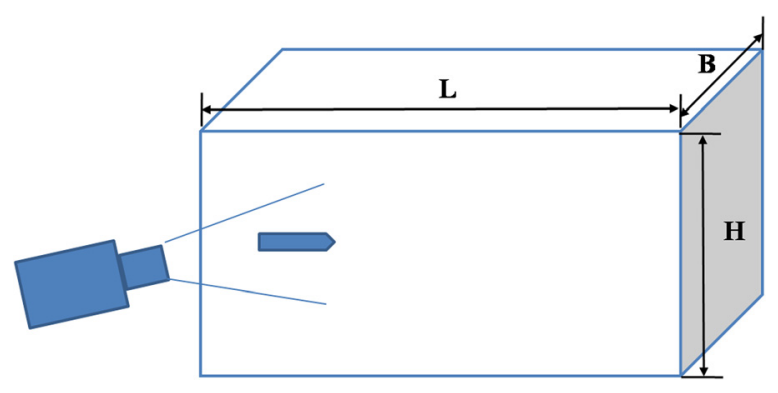

Fig. 2 Schematic diagram of water tank 


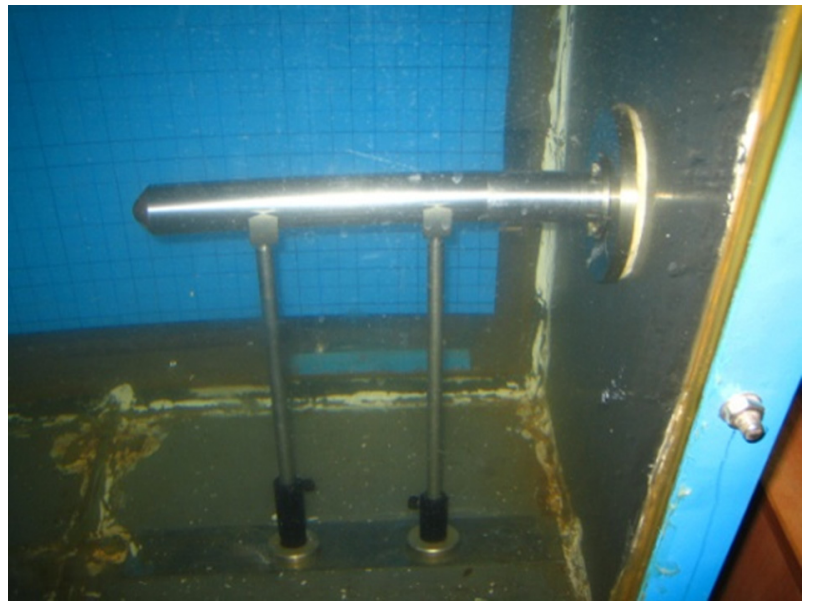

Fig. 3 Experimental model in water tank

Given that the experimental duration is less than $0.1 \mathrm{~s}$, the projectile runs in a quasi-horizontal track, and the cavity generated around the shoulder of the projectile is axisymmetric.

2.4 Typical Cavity Photograph. As shown in Fig. 4, a photograph of typical cavitation can be obtained using a high-speed camera with $10^{5} \mathrm{fps}$. The shoulder and tail cavities with shedding bubbles can be seen clearly. The length and thickness of the cavity are measured, as shown in Fig. 4. The precision of the length and thickness is approximately a pixel of the image, which stands for $0.64 \mathrm{~mm}$. In Secs. 3 and 4, the evolution of shoulder cavities will be mainly discussed on the basis of experimental pictures and numerical results.

\section{Numerical Methods}

3.1 Two-Dimensional Method With Mixture and RANS Approaches. To simulate the motions and phase change of liquid water and vapor, mixture/multiphase flow equations are adopted on the basis of the third type of method, as mentioned in Sec. 1. The continuity and momentum equations for the mixture of liquid water, vapor, and noncondensable air are established as

$$
\begin{gathered}
\frac{\partial}{\partial t}\left(\rho_{m}\right)+\nabla \cdot\left(\rho_{m} \mathbf{v}_{m}\right)=0 \\
\frac{\partial}{\partial t}\left(\rho_{m} \mathbf{v}_{m}\right)+\nabla \cdot\left(\rho_{m} \mathbf{v}_{m} \mathbf{v}_{m}\right) \\
=-\nabla p+\nabla \cdot\left[\left(\mu_{m}+\mu_{t}\right)\left(\nabla \mathbf{v}_{m}+\nabla \mathbf{v}_{m}^{\mathrm{T}}\right)\right] \\
+\rho_{m} \mathbf{a}
\end{gathered}
$$

where $p$ is the mixture pressure, $\rho_{m}$ is the mixture density, $\mathbf{v}_{m}$ is the mixture velocity vector, and $\mathbf{a}$ is the acceleration of the flow field. The laminar viscosity $\mu_{m}$ is defined as a density-weighted average of the three components. $\mu_{t}$ is the turbulent viscosity closed by RNG $k-\varepsilon$ model. The mixture density $\rho_{m}$ is defined by

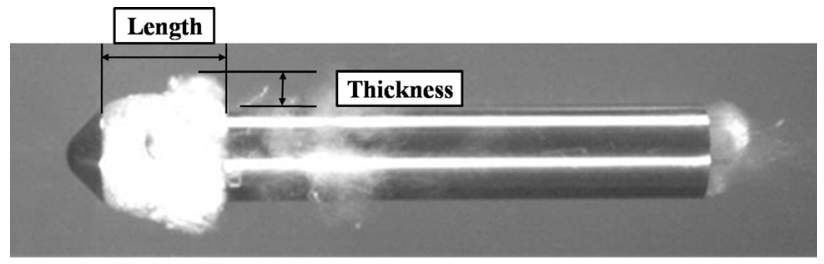

Fig. 4 Typical cavitation photograph

$$
\frac{1}{\rho_{m}}=\frac{f_{v}}{\rho_{v}}+\frac{f_{\mathrm{ncg}}}{\rho_{\mathrm{ncg}}}+\frac{1-f_{v}-f_{\mathrm{ncg}}}{\rho_{l}}
$$

where $f_{v}$ and $f_{\text {ncg }}$ are the component mass fractions, and $\rho_{v}, \rho_{\text {ncg }}$, and $\rho_{l}$ are the component densities of the vapor, noncondensable gas, and liquid components, respectively. During calculation, $f_{\text {ncg }}$ is assumed to be a small constant as $15 \mathrm{ppm}$.

The mass fraction equation for vapor is

$$
\frac{\partial}{\partial t}\left(\rho_{m} f_{v}\right)+\nabla\left(\rho_{m} \mathbf{v}_{m} f_{v}\right)=R_{e}-R_{c}
$$

where $R_{e}$ and $R_{c}$ are the evaporation and condensation rates, respectively, which can be simulated in the cavitation model established by Singhal et al. [6]

$$
\begin{cases}R_{e}=C_{e} \frac{\sqrt{k}}{\gamma} \rho_{l} \rho_{v} \sqrt{\frac{2\left(p_{v}-p\right)}{3 \rho_{l}}}\left(1-f_{v}-f_{g}\right), & p<p_{v} \\ R_{c}=C_{c} \frac{\sqrt{k}}{\gamma} \rho_{l} \rho_{l} \sqrt{\frac{2\left(p-p_{v}\right)}{3 \rho_{l}}} f_{v}, & p>p_{v}\end{cases}
$$

In this model, $\gamma$ is the coefficient of surface tension; $k$ is the turbulence kinetic energy; and $C_{e}$ and $C_{c}$ are the two empirical coefficients set as 0.02 and 0.01 in the following simulation, respectively.

To calculate the turbulent viscosity $\mu_{t}$ in Eq. (3), two transport equations for the turbulence kinetic energy $k$ and its dissipation rate $\varepsilon$ are solved as RNG $k-\varepsilon$ model, i.e.,

$$
\begin{gathered}
\frac{\partial}{\partial t}\left(\rho_{m} k\right)+\frac{\partial}{\partial x_{i}}\left(\rho_{m} k v_{i}\right)=\frac{\partial}{\partial x_{j}}\left(\alpha_{k} \mu_{\mathrm{eff}} \frac{\partial k}{\partial x_{j}}\right)+G_{k}-\rho_{m} \varepsilon \\
\frac{\partial}{\partial t}\left(\rho_{m} \varepsilon\right)+\frac{\partial}{\partial x_{i}}\left(\rho_{m} \varepsilon v_{i}\right)=\frac{\partial}{\partial x_{j}}\left(\alpha_{\varepsilon} \mu_{\mathrm{eff}} \frac{\partial \varepsilon}{\partial x_{j}}\right)+C_{1 \varepsilon} \frac{\varepsilon}{k} G_{k}-C_{2 \varepsilon} \rho_{m} \frac{\varepsilon^{2}}{k}
\end{gathered}
$$

where $G_{k}$ represents the generation of turbulence kinetic energy caused by the mean velocity gradients, calculated as $G_{k}=-\rho \overline{u_{i}^{\prime} u_{j}^{\prime}}\left(\partial u_{j} / \partial x_{i}\right)$. The quantities $\alpha_{k}$ and $\alpha_{\varepsilon}$ are the inverse effective Prandtl numbers for $k$ and $\varepsilon \alpha_{k}=\alpha_{\varepsilon}=1.393$, respectively. The model constants $C_{1 \varepsilon}=1.42$ and $C_{2 \varepsilon}=1.68$.

For an ordinary case, the turbulent viscosity is calculated by $\mu_{t}=C_{\mu} \rho_{m}\left(k^{2} / \varepsilon\right)$, which is overestimated in the mixed region [30]. Therefore, a modified turbulent viscosity is defined, which improves the simulations of the cloud shedding significantly. The turbulent viscosity is modified as $\mu_{t}=f(\rho) C_{\mu}\left(k^{2} / \varepsilon\right)$, where $C_{\mu}$ $=0.0845$, and $f(\rho)=\rho_{v}+\left(\left(\rho_{m}-\rho_{v}\right)^{n}\right) /\left(\left(\rho_{l}-\rho_{v}\right)^{n-1}\right), n=10$.

Unsteady numerical simulations are performed on the basis of finite-volume method with SIMPLEC scheme by using the commercial CFD software FLUENT. The version of FLUENT is 6.3. The equations are discretized by a second-order implicit scheme in time and a second-order upwind scheme in space. The numerical parameters are summarized in Table 1.

The computational domain, which is shown in Fig. 5, is discretized with a block-structured grid. The height of the first layer is

Table 1 Numerical parameters

\begin{tabular}{ll}
\hline \hline Simulation type & \multicolumn{1}{c}{ Unsteady } \\
\hline Pressure-velocity coupling & SIMPLEC \\
Temporal scheme & Second-order implicit \\
Time step & $5 \times 10^{-6} \mathrm{~s}$ \\
Spatial scheme & Second-order upwind \\
Pressure interpolation scheme & Body force weighted \\
Turbulence model & Modified RNG $k-\varepsilon$ \\
\hline
\end{tabular}




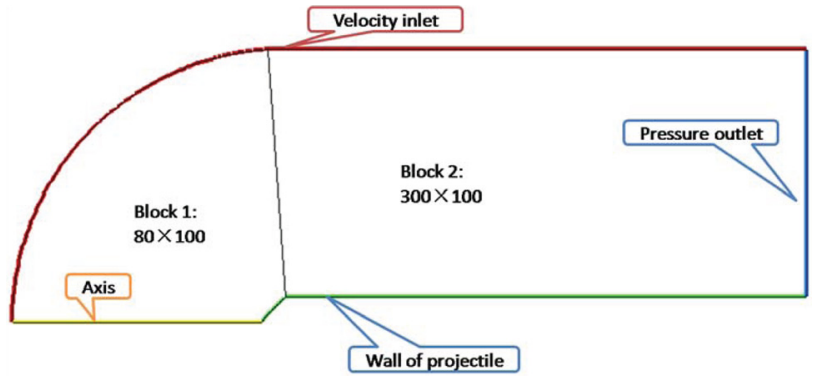

Fig. 5 Computational domain and mesh

set as $1 / 10,000$ of the projectile diameter to ensure $y^{+}$equals to one approximately. Thus, the two-layer model is adopted to resolve the laminar sublayer.

For the velocity inlet boundary condition, the inlet velocity is set as $18.5 \mathrm{~m} / \mathrm{s}$, the turbulent intensity is set as $1 \%$, and the hydraulic diameter is set as $0.037 \mathrm{~m}$. Therefore, the inlet $k$ and $\varepsilon$ can be calculated as 0.045 and 0.56 , respectively.

3.2 Three-Dimensional Method With VOF and LES Approaches. For comparison, LES method is also adopted in this study to show more detail of the flow patterns. The Navier-Stokes equations of incompressible flow by applying the filter function of LES are as follows:

$$
\nabla \cdot(\rho \overline{\mathbf{v}})=0
$$

$$
\frac{\partial}{\partial t}(\rho \overline{\mathbf{v}})+\nabla \cdot(\rho \overline{\mathbf{v}} \mathbf{v})=-\nabla \bar{p}+\nabla \cdot(\overline{\mathbf{S}}-\mathbf{B})
$$

where the overbar denotes the low-pass-filtered dependent variables as $\bar{\psi}=\int_{-\infty}^{+\infty} \psi G\left(x, x^{\prime}\right) d x^{\prime} . G\left(x, x^{\prime}\right)$ is the filter function; the top-hat filter function is adopted in this study.

$\overline{\mathbf{S}}=2 \mu \overline{\mathbf{D}}$ is the filtered viscous stress tensor, $\overline{\mathbf{D}}=1 / 2(\nabla \overline{\mathbf{v}}+$ $\left.\nabla \overline{\mathbf{v}}^{T}\right)$ stands for the filtered rate of stress tensor, and $\mu$ is the dynamic viscosity. $\mathbf{B}=(\overline{\mathbf{v v}}-\overline{\mathbf{v}})$, which means the subgrid stress tensor, represents the influence of small, unresolved eddies on the large, resolved ones.

On the basis of Boussinesq hypothesis, a subgrid viscosity $\mu_{\mathrm{SGS}}$ is considered as $\mathbf{B}=-2 \mu_{\mathrm{SGS}} \overline{\mathbf{D}}$. The entire viscous term can be described as $(\overline{\mathbf{S}}-\mathbf{B})=2\left(\mu+\mu_{\mathrm{SGS}}\right) \overline{\mathbf{D}}$, where $\mu_{\mathrm{SGS}}$ needs to be solved. In this study, $k-\mu$ subgrid scale model is applied to deal with subgrid stress

$$
\begin{gathered}
\frac{\partial k_{\mathrm{SGS}}}{\partial t}+\nabla \cdot\left(k_{\mathrm{SGS}} \overline{\mathbf{v}}\right)=\nabla \cdot\left[\frac{\mu+\mu_{\mathrm{SGS}}}{\rho} \nabla k_{\mathrm{SGS}}\right]+2 \frac{\mu_{\mathrm{SGS}}}{\rho} \overline{\mathbf{D D}}-C_{e} \frac{k_{\mathrm{SGS}}^{\frac{3}{2}}}{\bar{\Delta}} \\
\mu_{\mathrm{SGS}}=C_{k} \rho \bar{\Delta} \sqrt{k_{\mathrm{SGS}}}
\end{gathered}
$$

In the above equations, $\bar{\Delta}$ is the spatial filter size, $C_{e}=1.048$, and $C_{k}=0.094$.

$\rho, \mu$, and $p$ are the density, viscosity, and pressure of the mixture, respectively, i.e.,

$$
\begin{aligned}
& \rho=\alpha \rho_{l}+(1-\alpha) \rho_{v} \\
& \mu=\alpha \mu_{l}+(1-\alpha) \mu_{v}
\end{aligned}
$$

where $\alpha$ is the liquid volume fraction solved by VOF approach with a transport equation as

$$
\frac{\partial \alpha}{\partial t}+\nabla \cdot(\overline{\mathbf{v}} \alpha)=\frac{\dot{m}}{\rho_{l}}
$$

where $\dot{m}$, the mass transfer rate, is modeled by Kunz cavitation model [12]

$$
\begin{gathered}
\dot{m}^{+}=\frac{C_{v} \rho_{v} \alpha \min \left[0, \bar{p}-p_{v}\right]}{\left(1 / 2 \rho_{l} U_{\infty}^{2}\right) t_{\infty}} \\
\dot{m}^{-}=\frac{C_{c} \rho_{v} \alpha^{2}(1-\alpha)}{t_{\infty}}
\end{gathered}
$$

The open source code OpenFOAM is used with the secondorder implicit scheme for time discretization and Gauss linear interpolation for spatial discretization. The time step is adjustable with the maximum Courant number 0.5, and the average time step is approximately $0.1 \mu \mathrm{s}$. The $3 \mathrm{D}$ domain with hexahedral grids is adopted, as shown in Figs. 6 and 7. The cell number is approximately $38 \times 10^{6}$ with good orthogonality. Compared with meshes with several million elements, which are often used for similar cases [22,27], this mesh resolution is enough to present the major characteristics of unsteady behavior.

\section{Results and Discussion}

4.1 Unsteady Evolution of Shoulder Cavities. From the experimental and numerical results, cavity shapes evolve in three stages, namely, cavities grow (as shown in Fig. 8-stage 1),

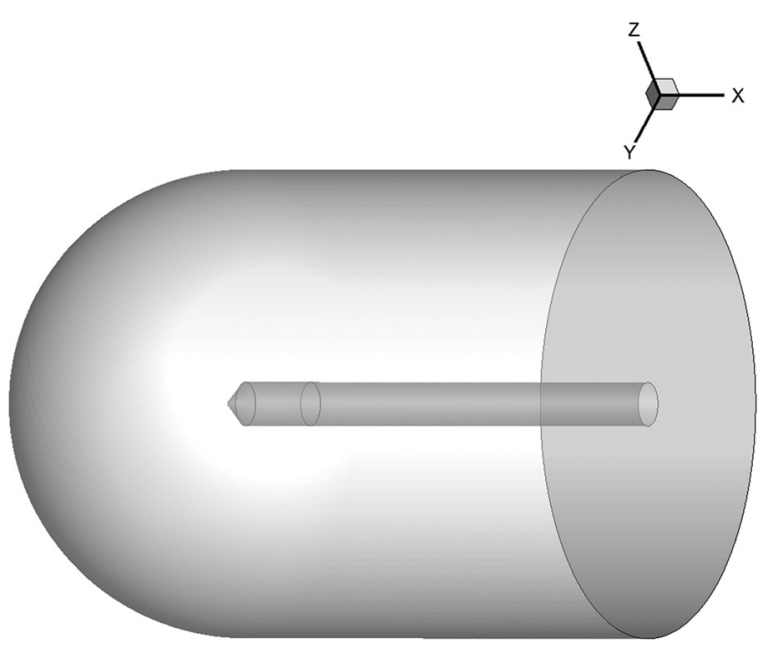

Fig. 6 Computational domain of the 3D method

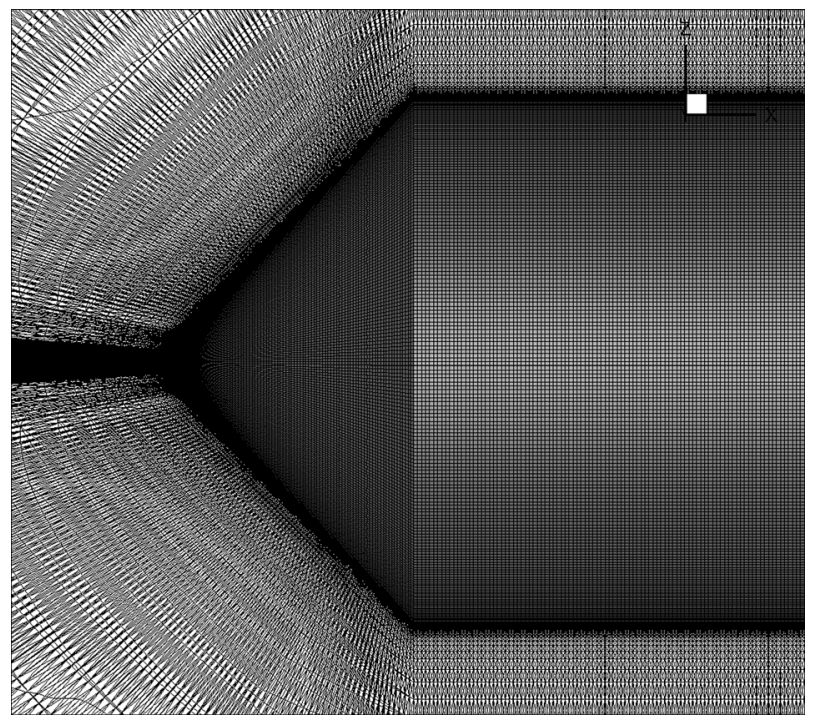

Fig. 7 Mesh near the head of the vehicle of the 3D method

Transactions of the ASME 


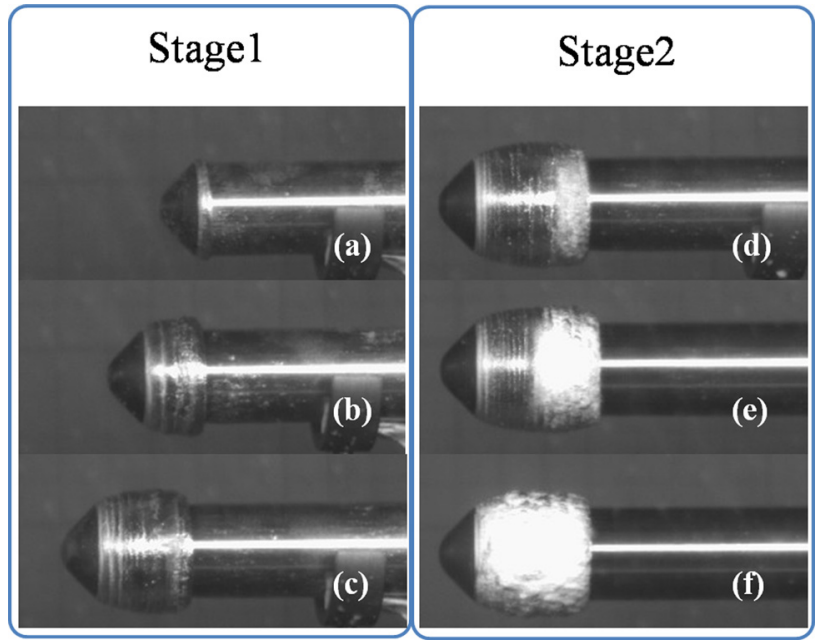

Fig. 8 Typical cavitation patterns in stage 1 and 2

re-entry jets develop and back to the shoulder (as shown in Fig. 8stage 2), and cavities shed quasi-periodically (as shown in Fig. 9). The re-entry jet is a liquid stream, which is opposite to the direction of the main flow in the cavity. Re-entry jet is produced by the adverse pressure gradient near the closure of the cavities. Given that the first and second stages have already been studied in previous works [27,31], the current investigation focuses on the evolutions in the third stage to investigate the strong unsteady behavior.

Figure 9 presents the eight successive shapes of the cavities obtained during each complete cavitation cycle. The left-side views are the photographs gained in the experiment. The middleside views show the volume fractions of the vapor phase obtained by $2 \mathrm{D}$ simulation. The right-side views show the isosurfaces, in which the vapor phase volume fraction is 0.1 in $3 \mathrm{D}$ results. In the pictures, $t^{*}$ represents the dimensionless time $t^{*}=t v_{0} / D$. The typical periodical time of the break-off cycle is about 3.7 both in experimental and numerical results. The shape variety of bubbles in the second cycle can be described as follows (as shown in Fig. 9):

(1) When the re-entry jet reaches the shoulder, cavitation bubbles collapse at the shoulder point. At this time, cavitation
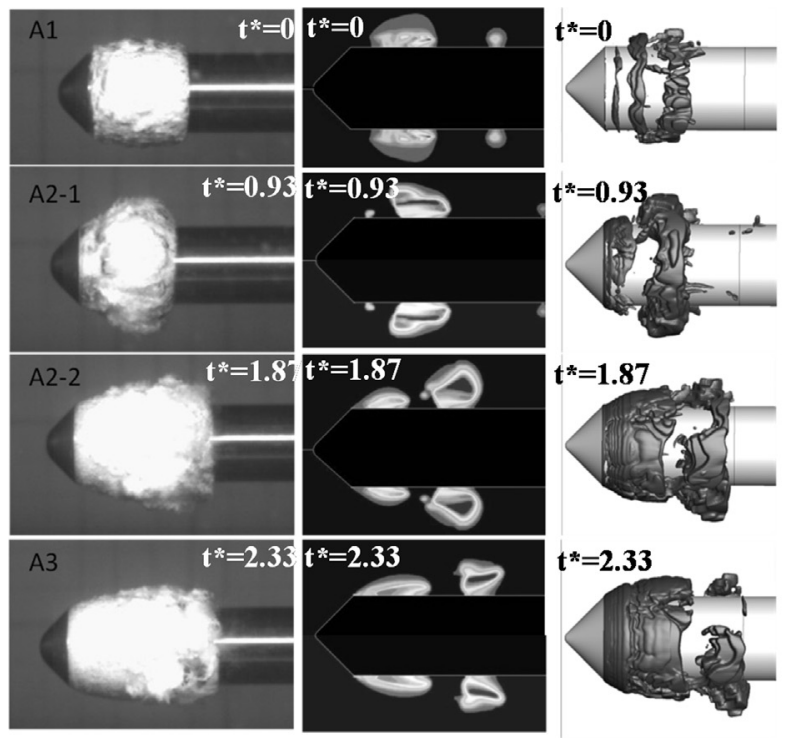

bubbles, which can be seen obviously, are referred to as remaining cavities (as shown in Fig. 9-A1).

(2) New cavities incept at the shoulder point and grow gradually. The remaining cavities mentioned above are drawn into the vortex area; they become long and thick, rotate, and shed downstream (as shown in Figs. 9-A2-1 and 9-A2-2).

(3) New cavities continue to grow longer. From the vortex intensity starting to diminish, the remaining cavities become increasingly thin (as shown in Fig. 9-A3).

(4) The shedding remaining cavities shrink and collapse finally, while new re-entry jet forms (at the location the red arrow pointing to, as shown in Fig. 9-A4).

(5) Re-entry jet moves upstream and crosses with the main flow, causing the cavities to be divided into two parts and forcing the new cavities to be thin and short. As shown in Figs. 9-A5-1 and 9-A5-2, a gap exists (at the location the red arrow pointing to) between the two parts, which can be seen both in the experimental and numerical results. However, the gaps in the experimental and $3 \mathrm{D}$ results are less obvious than the $2 \mathrm{D}$ result, which may be because the thickness of re-entrant jet in the experiment is thinner than that in the 2D simulation. Because we cannot measure the thickness in the experiment, as a reference Callenaere et al. [5] reported that the ratio of the re-entrant jet thickness to the cavity thickness ranges between $15 \%$ and $35 \%$. The ratio for axisymmetric condition is thicker than that for 2D condition theoretically, but the quantitative measurement is still need to be performed in the future.

(6) Re-entry jet reaches the shoulder, and the upstream part of the cavities collapses at the shoulder point. This time is also equivalent to the first status of the subsequent cycle (as shown in Fig. 9-A6, also numbered as B1).

In this cycle, the time histories of the length and thickness of shoulder cavities are measured, as shown in Fig. 10. The deviation in the experimental results is $0.5 \mathrm{~mm}$, which corresponds to 1 pixel in the photographs. The simulated results of cavity lengths agree well with the experimental phenomena. The thickness of shedding cavities of 2D RANS results is slightly larger than that of the experimental results. However, the simulations of unsteady cavitating flow, which use the governing equations, including the cavitation and turbulence model used above, can reflect the

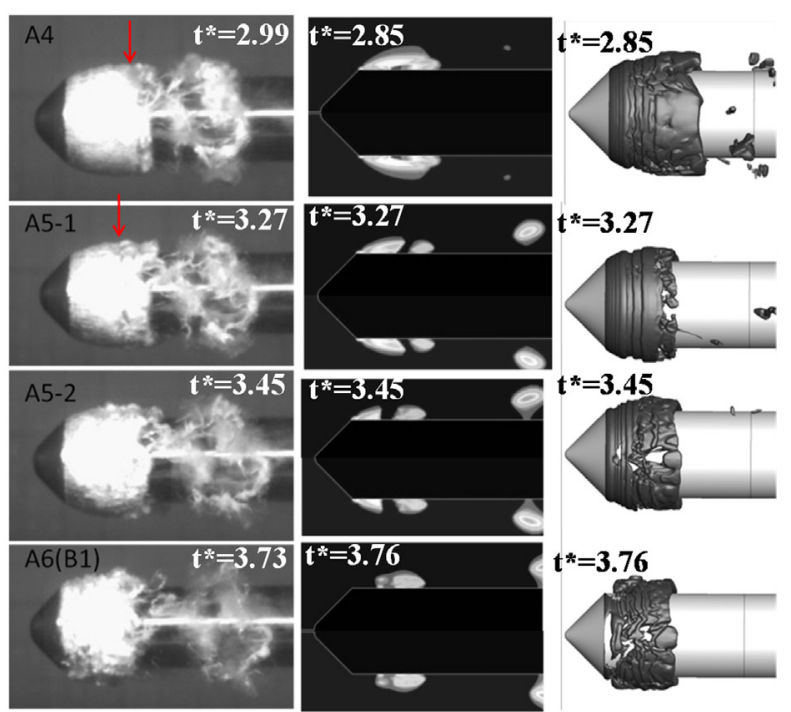

Fig. 9 Time evolution of cavitation patterns obtained from the experiment and simulation 


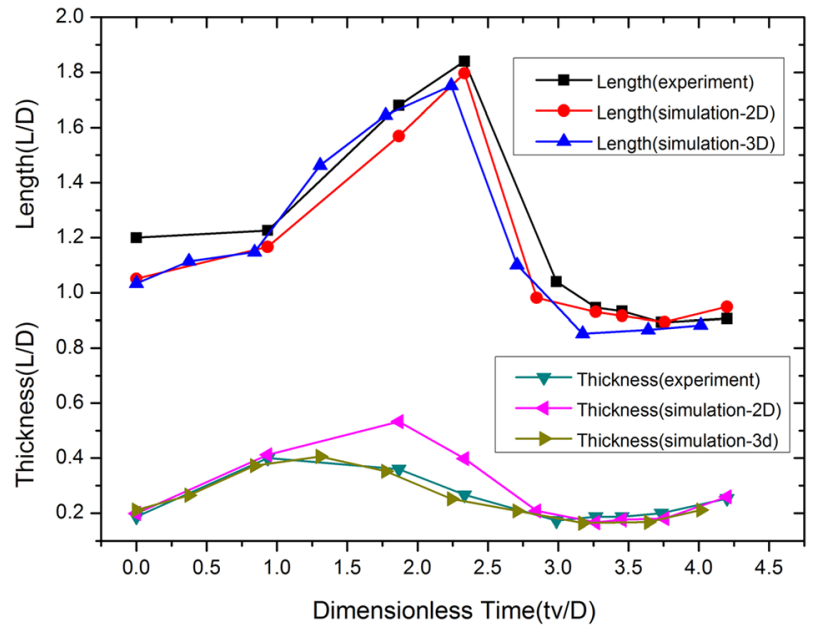

Fig. 10 Numerical and experimental results of cavity length and thickness physical phenomena effectively. Therefore, 2D simulation is effective to describe the general evolution of cavities.

In Sec. 4.2 , the $2 \mathrm{D}$ results are widely used to investigate the phenomena and mechanism of cavitation instability. The 3D results are only used to show the generation and evolution of vortices as comparison and supplementary, and the differences in shedding cavity simulation are analyzed.

4.2 Instability Induced by Re-Entry Jet. As mentioned above, the instability of cloud cavitation is induced mainly by the evolutions of re-entry jet, which is produced by the adverse pressure gradient near the closure of the cavities. The inception and development of re-entry jet can be analyzed by investigating the pressure and velocity around the wall of the projectile. Figures 11 and 12 show the time sequences of pressure and axial velocity distributions along a line paralleled to the axis, in which the distance between the line and $r$ axis is $0.51 D$. In the two figures, $x$-axis means the axial direction, and the origin of the coordinate is set at the theoretical point of the conical head. The dimensionless pressure $c_{p}$ and axial velocity $\tilde{v}$ are defined as $c_{p}=p / 0.5 \rho v_{0}^{2}$ and $\tilde{v}=v / v_{0}$, respectively, where $v_{0}$ is the speed projectile in this

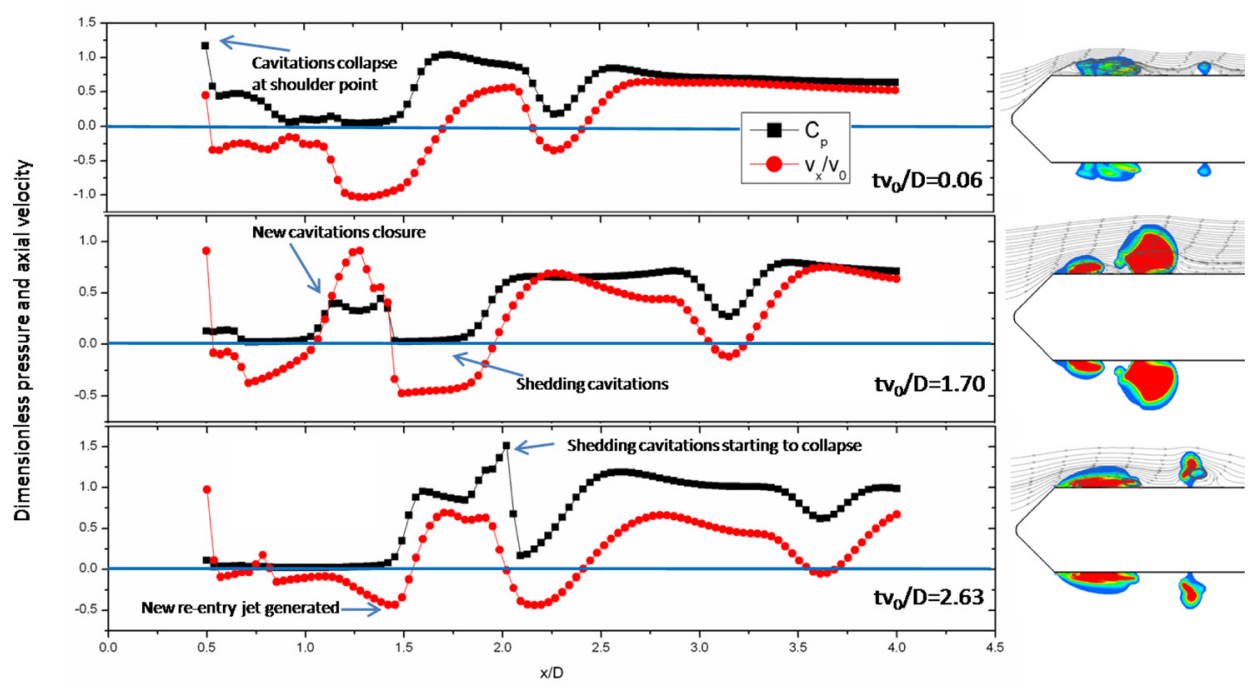

Fig. 11 Time sequence of pressure and velocity distributions along the line paralleled to the axis (1)

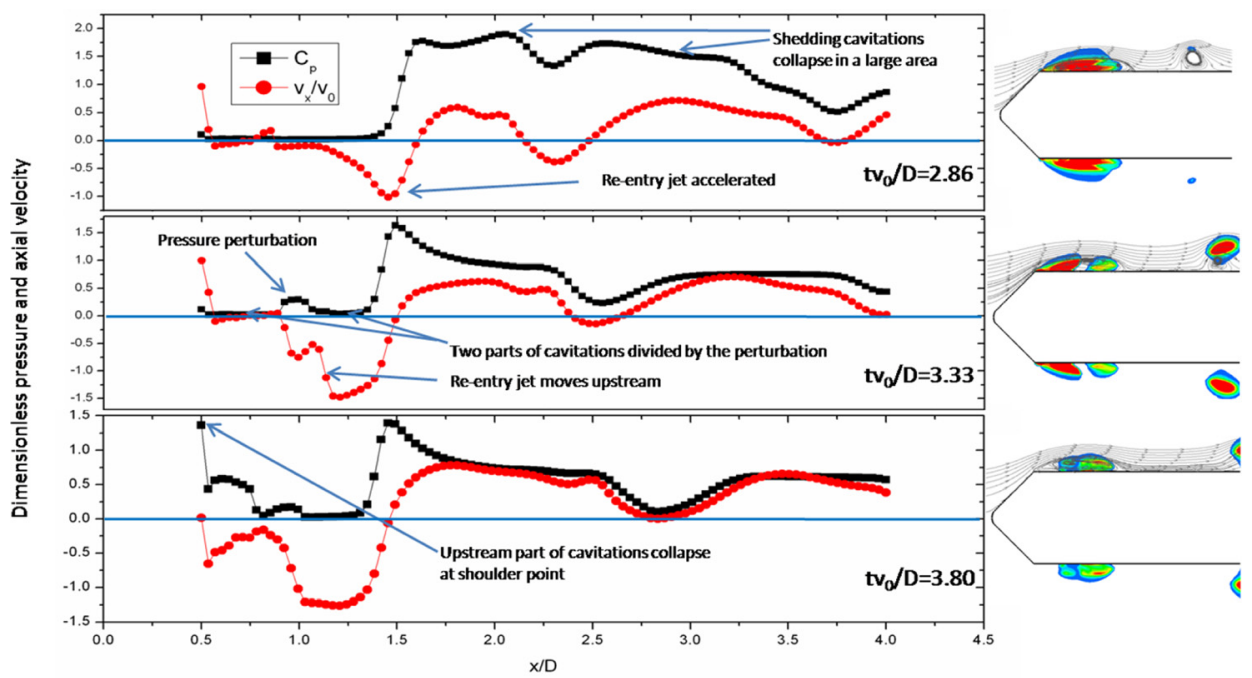

Fig. 12 Time sequence of pressure and velocity distributions along the line paralleled to the axis (2)

061102-6 / Vol. 138, JUNE 2016

Transactions of the ASME

Downloaded From: http://fluidsengineering.asmedigitalcollection.asme.org/ on 03/04/2016 Terms of Use: http://www.asme.org/about-asme/terms-of-use 
case, as mentioned previously. The re-entry jet can be shown in the velocity distribution curve (as shown in Figs. 11 and 12). It has negative $x$-velocity and its right boundary is located at the rear end of the sheet cavity, where the adverse pressure gradient is large.

The time sequences can be represented as follows:

(1) For the top view in Fig. 11, cavities collapse at the shoulder point, where a peak value of pressure exists. The re-entry jet generated in the last cycle still exists in the remaining cavities, which will react with the main flow to form a vortex.

(2) New cavities generate from the shoulder point. Correspondingly, the pressure at the new cavity closure gradually increases. The remaining cavities of the last cycle are controlled by the vortex, shedding downstream (as shown in the middle view in Fig. 11).

(3) Shedding cavities start to collapse, producing a pressure pulse near the cavity closure. Meanwhile, the new re-entry jet generates in the cavities near the closure by the adverse pressure gradient (as shown in the bottom view in Fig. 11).

(4) Shedding bubbles continue collapsing. They generate a high pressure in a large area, which increases the adverse pressure gradient and accelerates the re-entry jet significantly (as shown in the top view in Fig. 12).

(5) Re-entry jet moves upstream and produces a pressure perturbation inside the cavities, which divides the bubble into two parts (as shown in the middle view in Fig. 12).

(6) As the sustained movement of the re-entry jet, the upstream part of cavities shrinks rapidly to collapse at the shoulder point finally. At this moment, a cycle of cloud cavitation induced by the motion of re-entry jet is completed (as shown in the bottom view in Fig. 12).

The preceding analysis implies that the collapse of shedding cavities plays a vital role in the development and strength improvement of re-entry jet. Figure 13 shows the time history of the pressure coefficients of different points on the projectile wall. The pressure peaks generated by the cavity collapse (which are up to eight at shoulder point and two around shedding cavities, as shown in the top and bottom views in Fig. 13, respectively) are significantly higher than the pressure at the closure of the cavity as quasi-stagnation pressures (which are usually $0.3-0.5$, as shown in the middle view in Fig. 13). The results are similar with those reported by Iga et al. [32] in the cavitation surge phenomenon in cascade.

The pressure and velocity distributions at the same moments with Figs. 11 and 12 are shown in Fig. 14. From the pressure contour, the main and shedding cavities are all located in the lowpressure area, and a large area of high pressure is generated by the collapse of the shedding cavity in the down-left view of Fig. 14. As shown by the velocity vectors, the evolution of re-entry jet can be clearly seen inside the cavity. The re-entry jet is generated when the shedding cavity collapses and there is a large adverse pressure gradient at the end of the cavity. The re-entry jet is shown as the large velocity region inside the cavity pointed by the arrow (on the moments of 2.86, 3.33, and 3.80 shown in Fig. 14). And the jet thickness is approximately half of the cavity thickness. The shedding cavity always moves downstream with a vortex.

4.3 Interaction Between Vortices and Cavities. The contours of the turbulent kinetic energy of the 2D results are shown in Fig. 15. The lines represent the density variation in cavity area. Two main areas exist, where the turbulent kinetic energy is high; one area is around the shoulder where the flow accelerates, and the other area is near the wall between the main and shedding cavities. By contrast, the turbulent kinetic energy is often low inside the cavity. It also shows that the mixture density is between 500 and $900 \mathrm{~kg} / \mathrm{m}^{3}$ in the re-entry jet regions at moments of 2.86 , 3.33 , and 3.80, which indicates that the re-entry jet has a large component of liquid water (as shown in Fig. 15).

Compared with the contours in Fig. 15, the vorticity magnitude contours on the slices of the 3D results are shown in Fig. 16. Given that more scales of vortices can be resolved using LES method, the vorticity magnitude distribution in the cavitation region such as the high value areas is similar to that of the turbulent kinetic energy in the 2D results shown in Fig. 15. But the thickness in the $2 \mathrm{D}$ results is larger especially in the front conical region before the separation point of the projectile. In addition, the 2D RANS approach uses the homogeneous isotropic turbulence model; thus, the shedding vortices are more isotropic in different directions (as shown in Fig. 15, $t v_{0} / D=1.70$ ). By contrast, the shapes of shedding vortices in the $3 \mathrm{D}$ results are influenced by

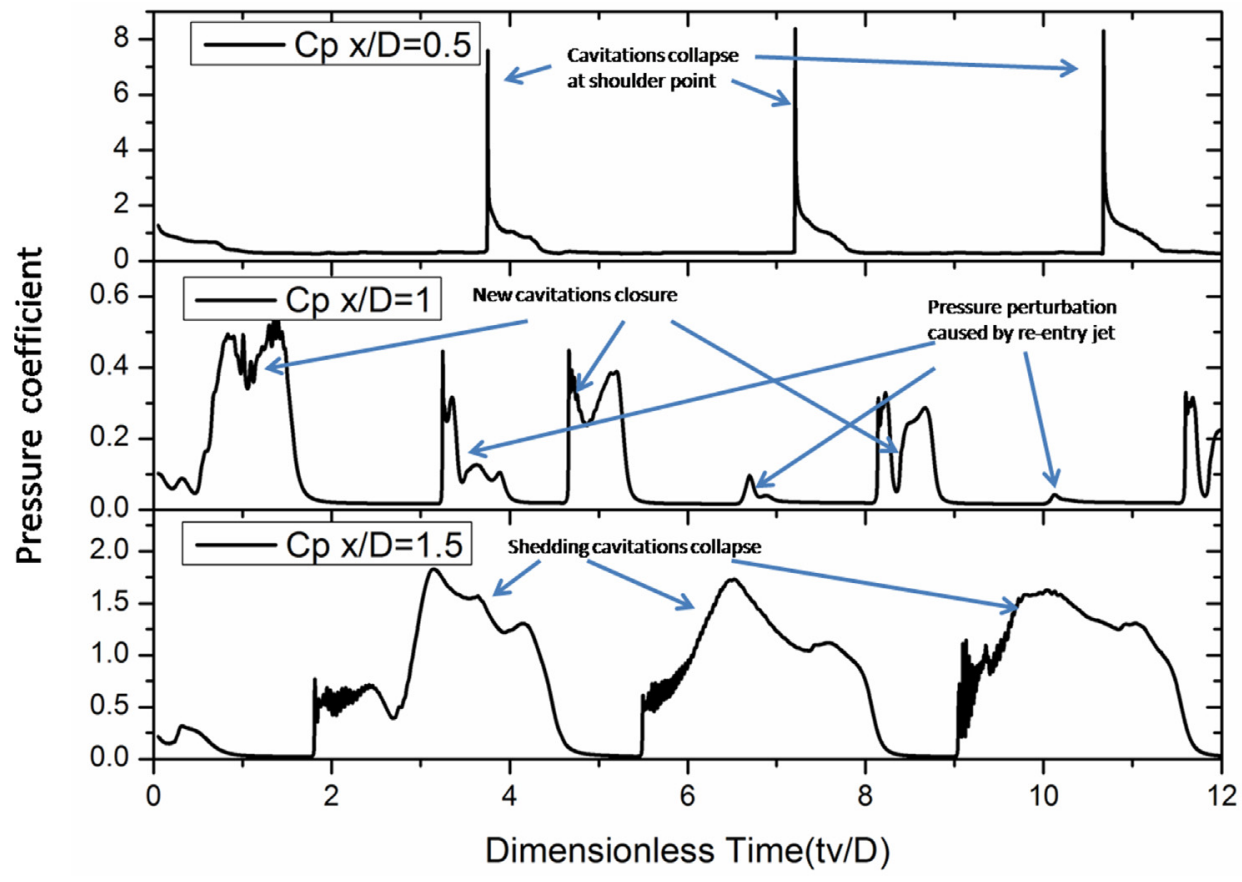

Fig. 13 Time history pressure coefficients at different points along the wall 

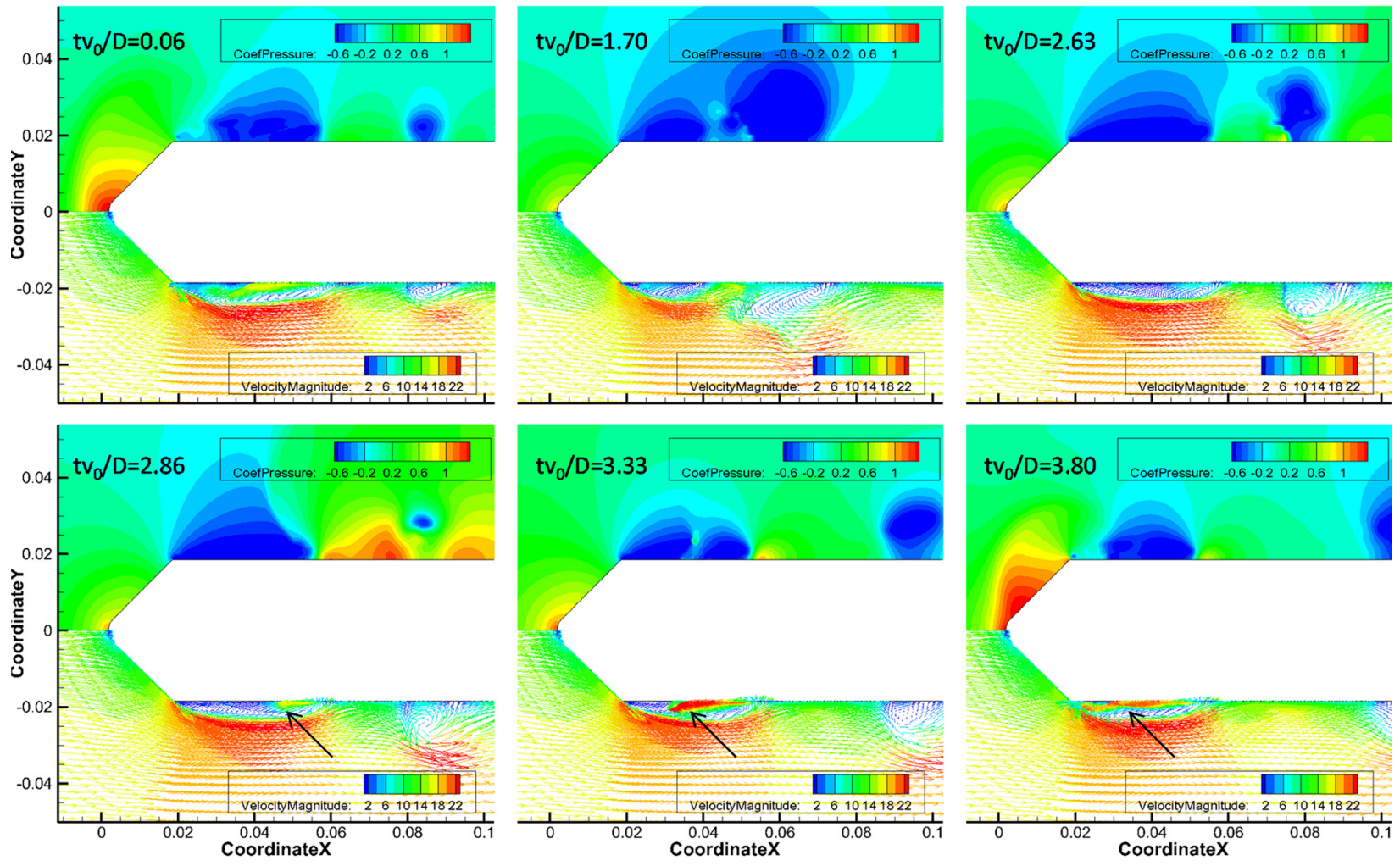

Fig. 14 Time sequence of pressure and velocity distributions in the flow field
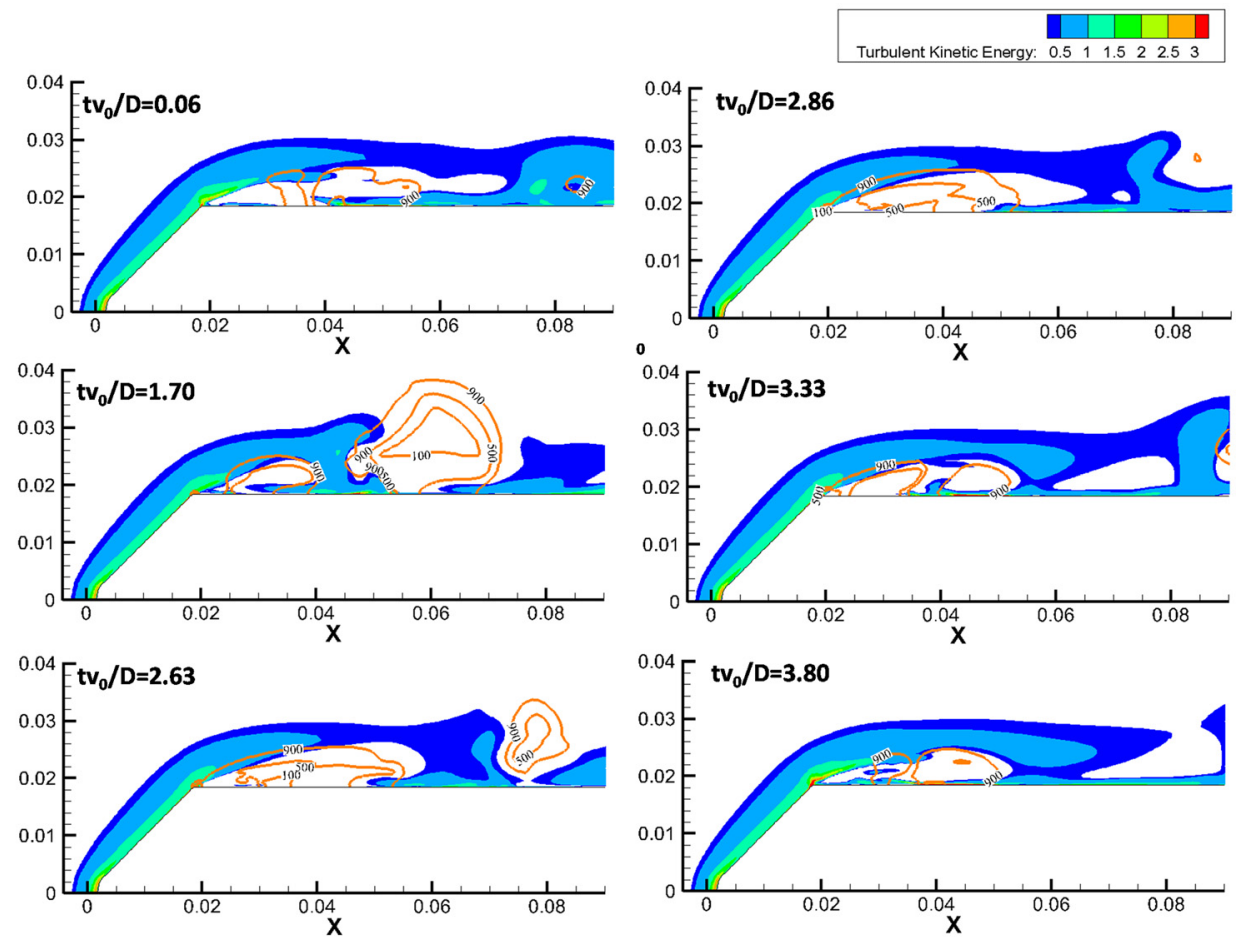

Fig. 15 Time sequence of turbulent kinetic energy contours

the main flow. The depression to the downstream limits the overall height of the vortex (as shown in Fig. 16-A2-2). The heights of the shedding cavities are also lower than the $2 \mathrm{D}$ results, which are more similar to the experimental observation. However, the 2D simulation can still obtain the important large-scale vortices well, which interact with the cavity evolution closely.
Besides the main vortices mentioned above, we can obtain more details in the 3D LES results. Figure 17 shows the time sequence of the isosurfaces, on which the vorticity magnitude is $5000 \mathrm{~s}^{-1}$, and the color stands for the axial velocity. In one cycle, the newly generated cavity around the shoulder is located in large-scale regular vortices, and insignificant perturbation occurs 


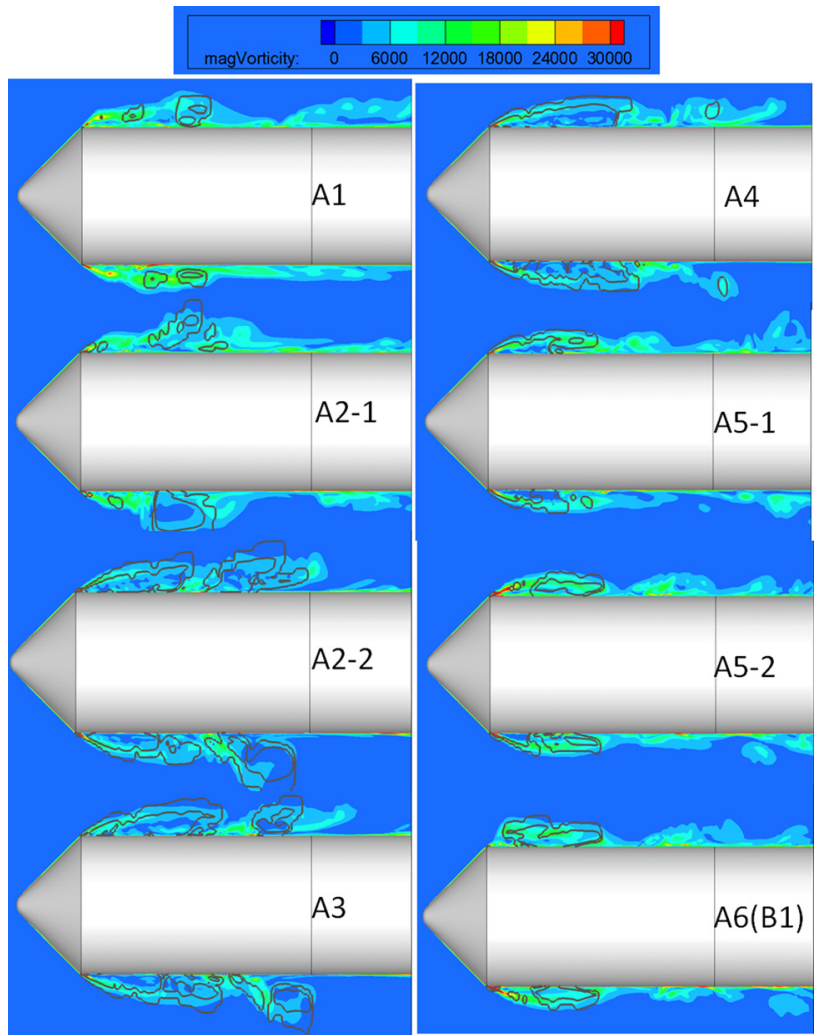

Fig. 16 Time sequence of vorticity magnitude contours on the slice of the $3 \mathrm{D}$ results by the fluctuation velocity (shown in Fig. 17-A1-A3). Consequently, the newly generated cavity often seems to be clean and smooth (shown in Fig. 9-A1-A3, also shown in Fig. 8-stage 1). The shedding cavities move between the main flow and re-entry jets with much perturbation by small vortices. After the shedding cavities collapse, the vortices are further broken down, carrying a small amount of gas downstream (shown in Fig. 17-A3-A5). New re-entry jets are then generated and flow upstream, breaking the vortices in the main cavity. The water and vapor are mixed sufficiently by the re-entry jet; hence, the cavity becomes irregular and rough (shown in Fig. 17-A5-A6, also shown in Fig. 8-stage 2).

\section{Conclusions}

In this study, an experimental system based on SHPB launching and high-speed photographing was established for cloud cavitation around slender axisymmetric projectiles. The corresponding phenomena were then predicted using two different types of numerical methods based on 2D mixture-RANS approach and 3D VOF-LES approach. The experimental and numerical results represent similar quasi-periodical unsteady evolutions of cavities near the shoulder.

The investigations of pressure and velocity evolutions show that the re-entry jet, which is produced and accelerated by the adverse pressure gradient, is the key factor to the cavitation instability. Pressure pulses generated by the cavity collapse are significantly higher than the pressure at the closure of the cavity as quasi-stagnation pressures. Thus, they play a vital role in the development and strength improvement of re-entry jet in this case.

The 2D method can represent the physical phenomena effectively, and the evolution of the main cavities gained with it agrees well with that in the 3D LES simulations. Therefore, the 2D method adopted in this study can be further used to predict the unsteady cavitation around vehicles with the similar shape in engineering applications. The 3D LES method can give better
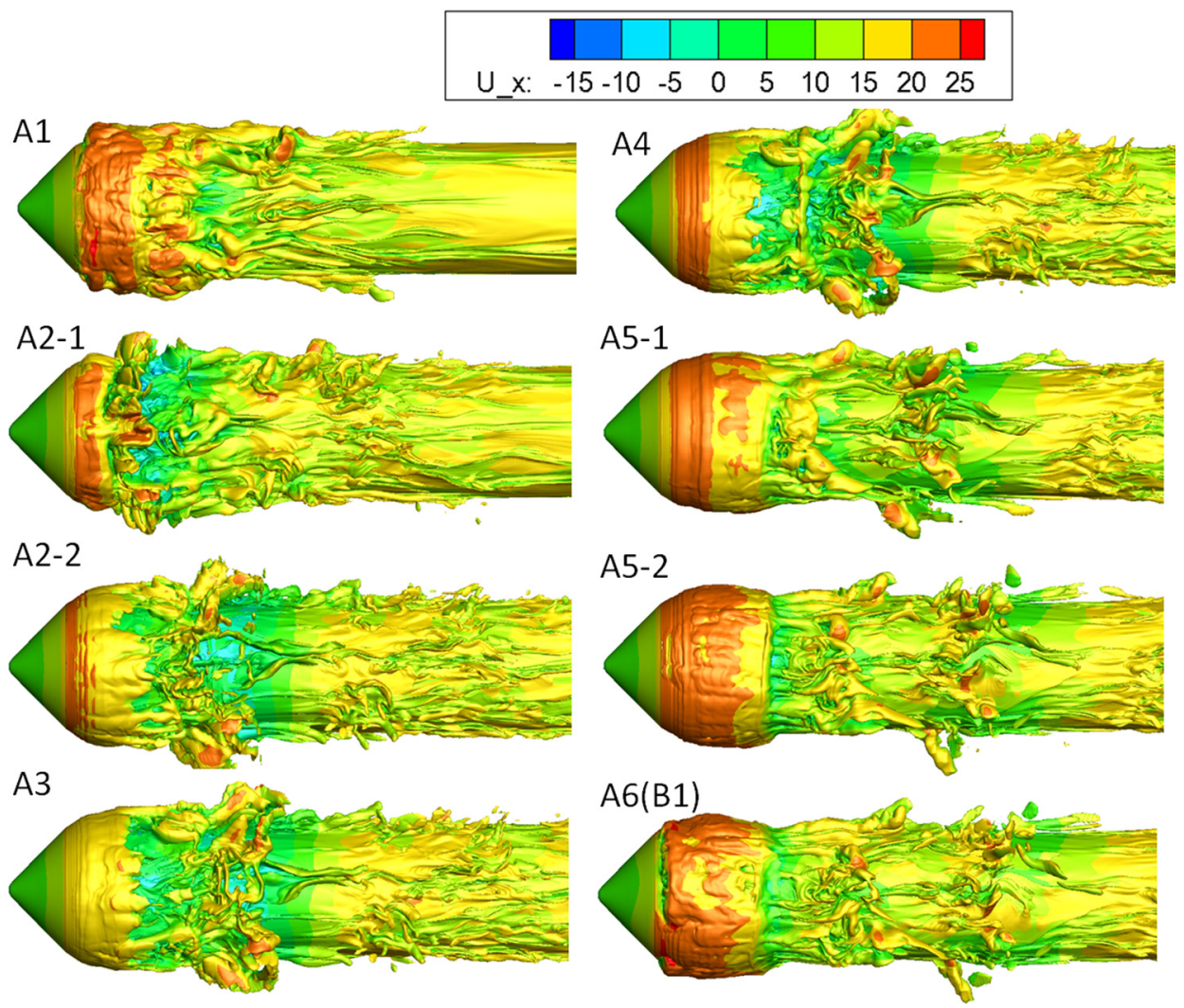

Fig. 17 Time sequence of the isosurfaces on which the vorticity magnitude is $5000 \mathrm{~s}^{-1}$ 
simulation on the shedding vortices than the 2D method, and more accurate shapes of shedding cavities are captured. Therefore, LES method is more effective for detailed analysis.

\section{Acknowledgment}

This research was sponsored by the National Natural Science Foundation of China under Contract Nos. 11332011 and 11202215 and the Youth Innovation Promotion Association of CAS (2015015).

\section{References}

[1] Brennen, C. E., 1995, Cavitation and Bubble Dynamics, Oxford University Press, New York, pp. 217-240.

[2] Stutz, B., and Legoupil, S., 2003, "X-Ray Measurements Within Unsteady Cavitation,” Exp. Fluids, 35(2), pp. 130-138.

[3] Stutz, B., and Reboud, J., 1997, "Experiments on Unsteady Cavitation," Exp. Fluids, 22(3), pp. 191-198.

[4] Stutz, B., and Reboud, J. L., 2000, "Measurements Within Unsteady Cavitation," Exp. Fluids, 29(6), pp. 545-552.

[5] Callenaere, M., Franc, J. P., Michel, J. M., and Riondet, M., 2001, "The Cavitation Instability Induced by the Development of a Re-Entrant Jet," J. Fluid Mech., 444, pp. 223-256.

[6] Singhal, A. K., Athavale, M. M., Li, H., and Jiang, Y., 2002, "Mathematical Basis and Validation of the Full Cavitation Model," ASME J. Fluids Eng., 124(3), pp. 617-624.

[7] Kunz, R. F., Boger, D. A., and Stinebring, D. R., 1998, "A Preconditioned Navier-Stokes Method for Two-Phase Flows With Application to Cavitation," Comput. Fluids, 29(8), pp. 849-875.

[8] Merkle, C. L., Feng, J., and Buelow, P. E. O., 1998, "Computational Modeling of the Dynamics of Sheet Cavitation," 3rd International Symposium on Cavitation, Grenoble, France, pp. 47-54.

[9] Arndt, R. E. A., 2002, "Cavitation in Vortical Flows," Annu. Rev. Fluid Mech., 34(1), pp. 143-175.

[10] Zhou, L., and Wang, Z., 2008, "Numerical Simulation of Cavitation Around a Hydrofoil and Evaluation of a RNG $\kappa-\varepsilon$ Model," ASME J. Fluids Eng., 130(1), p. 011302.

[11] Coutier-Delgosha, O., Stutz, B., Vabre, A., and Legoupil, S., 2007, "Analysis of Cavitating Flow Structure by Experimental and Numerical Investigations," J. Fluid Mech., 578, pp. 171-222.

[12] Coutier-Delgosha, O., Reboud, J., and Delannoy, Y., 2003, "Numerical Simulation of the Unsteady Behaviour of Cavitating Flows," Int. J. Numer. Methods Fluids, 42(5), pp. 527-548.

[13] Coutier-Delgosha, O., Fortes-Patella, R., and Reboud, J. L., 2003, "Evaluation of the Turbulence Model Influence on the Numerical Simulations of Unsteady Cavitation," ASME J. Fluids Eng., 125(1), pp. 38-45.

[14] Coutier-Delgosha, O., Deniset, F. O., Astolfi, J. A., and Leroux, J.-B., 2007, "Numerical Prediction of Cavitating Flow on a Two-Dimensional Symmetrical Hydrofoil and Comparison to Experiments," ASME J. Fluids Eng., 129(3), pp. 279-292.
[15] Wu, J. Y., Wang, G. Y., and Shyy, W., 2005, "Time-Dependent Turbulent Cavitating Flow Computations With Interfacial Transport and Filter-Based Models," Int. J. Numer. Methods Fluids, 49(7), pp. 739-761.

[16] Hu, C., Wang, G., Chen, G., and Huang, B., 2014, "A Modified PANS Model for Computations of Unsteady Turbulence Cavitating Flows," Sci. China Phys. Mech. Astron., 57(10), pp. 1967-1976.

[17] Ji, B., Luo, X., Arndt, R. E. A., and Wu, Y., 2014, "Numerical Simulation of Three Dimensional Cavitation Shedding Dynamics With Special Emphasis on Cavitation-Vortex Interaction," Ocean Eng., 87, pp. 64-77.

[18] Huang, B., Wang, G. Y., and Zhao, Y., 2014, "Numerical Simulation Unsteady Cloud Cavitating Flow With a Filter-Based Density Correction Model," J. Hydrodyn., 26(1), pp. 26-36.

[19] Huang, B., and Wang, G. Y., 2011, "Partially Averaged Navier-Stokes Method for Time-Dependent Turbulent Cavitating Flows," J. Hydrodyn., 23(1), pp. 26-33.

[20] Ji, B., Luo, X., Wu, Y., Peng, X., and Xu, H., 2012, "Partially-Averaged Navier-Stokes Method With Modified k-Epsilon Model for Cavitating Flow Around a Marine Propeller in a Non-Uniform Wake," Int. J. Heat Mass Transfer, 55(23-24), pp. 6582-6588.

[21] Bensow, R. E., and Bark, G., 2010, "Implicit LES Predictions of the Cavitating Flow on a Propeller," ASME J. Fluids Eng., 132(4), p. 041302.

[22] Ji, B., Luo, X. W., Arndt, R. E. A., Peng, X., and Wu, Y., 2015, "Large Eddy Simulation and Theoretical Investigations of the Transient Cavitating Vortica Flow Structure Around a NACA66 Hydrofoil,” Int. J. Multiphase Flow, 68, pp. 121-134.

[23] Wang, G., and Ostoja-Starzewski, M., 2007, "Large Eddy Simulation of a Sheet/Cloud Cavitation on a NACA0015 Hydrofoil," Appl. Math. Modell., 31(3), pp. 417-447.

[24] Huang, B., Zhao, Y., and Wang, G., 2014, "Large Eddy Simulation of Turbulent Vortex-Cavitation Interactions in Transient Sheet/Cloud Cavitating Flows," Comput. Fluids, 92, pp. 113-124.

[25] Dittakavi, N., Chunekar, A., and Frankel, S., 2010, "Large Eddy Simulation of Turbulent-Cavitation Interactions in a Venturi Nozzle," ASME J. Fluids Eng., 132(12), p. 121301.

[26] Roohi, E., Zahiri, A. P., and Passandideh-Fard, M., 2013, "Numerical Simulation of Cavitation Around a Two-Dimensional Hydrofoil Using VOF Method and LES Turbulence Model," Appl. Math. Model., 37(9), pp. 6469-6488.

[27] Yu, X., Huang, C., Du, T., Liao, L., Wu, X., Zheng, Z., and Wang, Y., 2014 "Study of Characteristics of Cloud Cavity Around Axisymmetric Projectile by Large Eddy Simulation,” ASME J. Fluids Eng., 136(5), p. 051303.

[28] Ji, B., Luo, X., Peng, X., and Wu, Y., 2013, "Three-Dimensional Large Eddy Simulation and Vorticity Analysis of Unsteady Cavitating Flow Around a Twisted Hydrofoil," J. Hydrodyn., 25(4), pp. 510-519.

[29] Wei, Y. P., Wang, Y. W., Fang, X., Huang, C. G., and Duan, Z. P., 2011, “A Scaled Underwater Launch System Accomplished by Stress Wave Propagation Technique," Chin. Phys. Lett., 28(2), p. 024601.

[30] Dular, M., Bachert, R., Stoffel, B., and Sirok, B., 2005, "Experimental Evaluation of Numerical Simulation of Cavitating Flow Around Hydrofoil," Eur. J. Mech. B/Fluids, 24(4), pp. 522-538.

[31] Wang, Y., Huang, C., Fang, X., Du, T., and Yu, X., 2013, "Characteristics of the Re-Entry Jet in the Cloud Cavitating Flow Over a Submerged Axisymmetric Projectile (in Chinese)," Chin. J. Hydrodyn., 28(1), pp. 23-29.

[32] Iga, Y., Hashizume, K., and Yoshida, Y., 2011, "Numerical Analysis of Three Types of Cavitation Surge in Cascade," ASME J. Fluids Eng., 133(7), p. 071102 . 